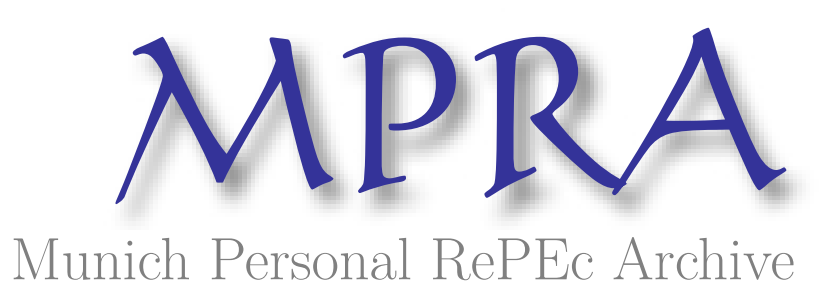

\title{
Economic Development and Efficiency Criteria in the Satisfaction of Basic Needs
}

Chichilnisky, Graciela

29 November 1976

Online at https://mpra.ub.uni-muenchen.de/7980/

MPRA Paper No. 7980, posted 30 Mar 2008 04:34 UTC 


\title{
Economic development and efficiency criteria in the satisfaction of basic needs*
}

\author{
Graciela Chichilnisky \\ Department of Economics, Harvard University, I737 Cambridge St, 40I, Cambridge. \\ Massachusetts 02/38, USA \\ (Received 29 November 1976; revised 14 March 1977)
}

\begin{abstract}
Introduction
Development and planning institutions that study the aggregate behaviour of the economy often use the value of the Gross National Product (GNP) as a primary criterion of growth. GNP is computed as the sum of the value of the total market production of final goods and services at market prices. However, in much of the underdeveloped world, scarcity of total production of certain basic goods (relative to survival values, say, of consumption of food, and minimal housing, health and educational requirements of the population) is a factor in welfare which is not necessarily adequately represented in GNP computations $^{b}$ : GNP values. may not provide adequate measures of other important economic variables. It is the hope of governments or decision makers in many underdeveloped countries that a goal of maximization of GNP be concomitant or consistent with the attainment of adequate levels of per capita consumption of basic goods. However, when income
\end{abstract}

\footnotetext{
- The author deyeloped this model while working for Fundacion Bariloche, Buenos Aires and Rio Negro. Argentina during the pericod 1972 74. It was used as a mathematical-economic basis for the subsequent empirical and computational work of the Latin American world model in a modified form. This paper contains results first presented at a meeting of the International Institute for Applied Systems Analysis in Vienna. October 1974 and also further work.

Discussions with Kenneth Arrow, Amilcar Herrera, Y. C. Ho, Helio Jaguaribe. Tjallings Koopmans. Carlos Mallman. Enrique Oteiza, and Jorge Sabato were very helpful in the construction and formalization of the model.

"Much of the production of underdeveloped countries that does not trade in the modern monetary market is not computed. For instance. food production and home buiding done and consumed by families. and labour exchanged without monetary payment, which can be an important part of total economic activity in so-called 'subsistence economies:

h GNP is computed by adding the monetary value of goods and services: the sum is made irrespective of the composition. For instance. if the value of an air conditioner system is 100 times the value of a pair of shoes, 100 shoes and $I$ air cunditioner would add equally.
}

distribution considerations are made, when the country has scarce resources for the production of alternative goods, and, in addition, when the market mechanism (local or international) is not working adequately, this hope may not materialize. There is evidence that this is the case in many underdeveloped countries ${ }^{c}$. at least. in the short- or medium-term horizon. Thus, it is of interest to examine alternative feasible growth path's and alternative criteria of economic efficiency of * growth. and to compare economic performance according to different measures, especially in countries where these problems are more pressing.

This paper aims to give a possible mathematical formalization of economic efficiency in an economy concerned with attaining acceptable levels of per capita consumption of basic goods, subject to economic and social welfare constraints. This model was constructed as an underlying mathematicaleconomic structure of the Latin American world model of Fundacion Bariloche. It has been used, in a modified form, as a feasibility study of possible growth paths in such underdeveloped economies. The model has a methodological intersection with the theory of optimal economic growth, in that the efficient growth paths are solutions of a constrained optimization problem: they optimize a social welfare criterion satisfying constraints given by a set of differential equations with initial values ${ }^{d}$. In the usual optimal growth models these differential equations represent production-investment-consumption relations through time, formalizing a trade-off between present and future consumption (more present consumption implies less present investment). Here, in addition. the admissible paths also satisfy a differential equation which relates the rate of population growth to the level of consumption of basic goods (a proxy for standard of living) which introduces further relationships between present and future consumption. The admissible paths are assumed to satisfy additional requirements. Per

\footnotetext{
"For studies on the question of income distribution and growth, see, for instance, H, Chenery et al. ${ }^{3}$

'See, for instance, Arrow and Kurz'
} 
capita consumption of all basic goods must be nondecreasing throughout time. This is a welfare consideration for countries with low levels of consumption. Also, the possible changes in the technology (relative capital labour intensities). and the relative investment of factors (capital and labour) in the different sectors of the economy has to vary within limits. These constraints represent friction and inertia of economic systems at high levels of aggregation.

The model has some other features that contrast with those of other existing global models". As discussed above. the social welfare function formalizes the objective of reaching, in an efficient manner. subject to the constraints, an acceptable level of consumption of basic goods. Efficiency is defined here in terms of the minimization of the time horizon at which these ucceptable consumption levels are reached. The model concerns the supply side of the economy, since it attempts to provide an alternative measure of efficiency in production and a basis for an empirical feasibility study for reaching such consumption levels. For a complete description of a market economy, the demand side and the relationships between supply and demand along the dynamic equilibria paths should be analysed. Such analysis could be used to contrast efficiency of market induced growth paths and paths oriented towards the achievement of the goals discussed here*. Because of its optimality or efficiency criteria, the model contrasts with other global models which basicatly simulate through extrapolation or projection. present trends into the future for the relevant economic variables ${ }^{f}$. Some form of rationalization of the use of scarce resources to satisfy basic needs is required in this model. Such a rationality may or may not be consistent with market behaviour, and it may require taxation or other public policies to be attained: further work on this point would be of interest. As discussed above, the growth of the population is assumed here to be partly dependent on per capita consumption. It turns out that empirically tested functions of demographic rates of growth dependent on economic variables ${ }^{z}$ are not necessarily always increasing functions of consumption: at some (higher) levels of consumption they are sometimes decreasing. The economic productive system, in turn. feeds from the demographic system. since availability of labour is restricted by the total (productive) population. Restricted substitution between capital and labour is allowed in production. For instance, at relatively high levels of the population variable. and relatively low levels of the capital

\footnotetext{
"Another limitation to the model is the lack of is theoretical incernational sector. This sector is of particular importance for developing countries: it has been simulated by different exogeneousily-given international rade patrerns in the empincal model:

'Set, for instance Meadows et ai." Part of the purpose or the Fundacion Bariloche model was to present an alternative approach 10 global modelling with a capacity for adjustment of the economic and demographic variables from within and not only a model projecting into the future present andior past economic or demographic tendencies. The simulation model built from this one attempts also to reproduce mechanisms of adjustments - as they appeared in the recent historical data, and simulate their eficcts in future persods. as well as compare those wath other policies ${ }^{5}$
}

"Derned from a demographic econometrte submodel: Herrera et al. variables, the model efficiency criterion may call for more labour-intensive technologies. This contrasts with other models where substitution between capital and labour is. in effect. not allowed: this being formalized by fixed capital/output and capital/labour ratios ${ }^{\text {h }}$.

Since there is some choice of technology in the different sectors, even though restricted, consumption and production are related to population growth, sometimes in opposite ways. This poses trade-offs through time between more (present) consumption (and perhaps lower population growth and hence increased future per capita consumption), and more investment (and hence more future productive capability), and between a selection of alternative technologies and future consumption.

The market prices enter into the empirical model as an important parameter. However, the values of per capita consumption of basic goods and the minimum requirements of consumption are not expressed in monetary value. but in real terms.

A brief description of the dynamics of the model in a sequential form can be given as follows. Initial values of production, consumption, and investment in three basic sectors are given inutrition, housing, health and education). and in a fourth sector of services and consumption goods complementary to the production and consumption of the three basic goods. Also. initial capital and labour allocations and production functions dependent on capital and labour, are given one for each sector. At each point in time a distribution of the available capital and labour for each of the production functions of the five basic sectors is chosen within a feasible set. A choice is also made at each period between investment and consumption of each sector. The optimization process aims at steering the economy along a path in which minimum acceptable levels of per capita consumption of the basic goods are attained in the shortest possible time horizon, so that the model concerns countries where these levels have not yet been reached. The demographic submodel of the population is fed from the values of the economic variables. This, in turn. determines the labour force variable. which is used in the constrained optimization for the choice of alternative capital-]abour mixes or technologies to be used next in the different sectors.

Below and in the appendix a description is given of the model and of how it was utilized in the Latin American world model ${ }^{6}$. In another section we study the existence and characterizations of the solution(s). and briefly discuss how this characterization cah be used to compare a given pattern of growth (for

\footnotetext{
"For instance, in Meadows et al. ${ }^{5}$ the assumption of a fixed cupital ioutpur ratio, together with the assumption of an exogeneous Iconstant) rate of population growth has the effect that the binding factors of productions are always capital and natural resources. Wish an funbounded population growth which is also unadaptive in the margin at each point in time. capital ends up being the only productive factor. People only consume, and finite natural resources hound growth when they are depleted. By its own structure, such a modet contains a carasirophe. In this model, by contrast. some adaptation of the economy to the relative avalability of scarce resources does occur. This is reminiscent of some of the neoclassicis literature on exploitation of natural resources: in those models the adaptation is brought about by adjustments of consumption to market prices. An analogous argument could be used here if the demand side was also considered.
} 
instance, one in which GNP is maximized) with the efficient paths of growth defined in this model.

\section{The model and its dynamics}

This section contains a description of the model and of its utilization in the Latin American world model. henceforth called the empirical model ${ }^{16}$.

Each economy for block of economies) is divided into four sectors:

$S_{1}$. food products: $S_{2}$, housing: $S_{3}$, education and health: and $S_{4}$, other services and consumption goods which are complementary to the production and consumption of goods and services of sectors $S_{1}, S_{2}$ and $S_{3}$. Consumption of sectors $S_{1}$ and $S_{3}$ is measured in certain physical standard units. For example, in the empirical model consumption of $S_{1}$ is measured in numbers of calories and in $S_{3}$ in the number of people that can be educated and provided with health care. Sector $S_{2}$ s output of housing is measured by the number of standard housing units or dwellings.

There are no corresponding units to measure consumption of the output of sector $S_{4}$; it was given in the empirical model in monetary value. It was also assumed that the value of the output of $S_{4}$ (in adequate prices) was roughly half of the value of total output. This hypothesis is consistent with available data across countries.

Each sector has a production technology that uses as inputs capital and labour; the technology of the $i$-th sector is described by a production function:

$$
F^{i}=R^{s} \rightarrow R^{*}
$$

where $F^{i}\left(L_{i}, K_{1 i}, \ldots K_{4 i}\right)$ represent the output of the $i$-th sector. $L_{1}$ denotes allocation of labour to sector $i$. and $K_{j i} \in R^{+}$denotes the allocation of type $j$ capital good to sector $i$. There are given initial values of capital stocks for each of the sectors of the economy, denoted $K_{i}(0) \in R^{+}, i=1, \ldots, 4$, determining an initial capital stock vector $K_{0}$. and an initial amount of total productive population denoted $L_{0} \in R^{+}{ }^{10}$ Total productive population at time $t \geqslant 0$ is denoted $L(t)$. and the total amount of capital goods of type $j$ available at time $t$ is denoted $K,(t) \in R^{+}$, so that:

$$
\sum_{j=1}^{4} K_{j}(t) \leqslant K_{i}(t)
$$

and

$$
\sum_{i=1}^{4} L_{i}(t) \leqslant L(t)
$$

At each point in time, for sector $i$ of the economy, $i=1,3$ and 4 , it is assumed that the sum of the consumption goods produced. denoted $c_{1}(t)$ and the investment realized on capital goods of the $i$ sector

\footnotetext{
'For a discusston of the classification of countries into blocks in the Latin American world model, see Herrera $e t$ al. ${ }^{\circ}$ and the remarks at the end of this section. The sectors defined here do not concude with those used in reference $S$ : there. there is, in addition. a capital stock sector, which here is disaggregated in capital stock used in the production of the sectors $S_{1} \ldots . . S_{4}$. see equations (I) and 13).

The demographic submodel of Herrera et al." gives a pyramid of population iby ages) at each time period. productive population is a proportion of cotal population.
}

(which is, by definition. the rate of change of type $j$ cupital stock $\hat{K}_{i}(t)=\left(\hat{c}_{i}(\hat{c} t) K_{i}(t)\right)$ does not exceed the productive capabilities of that sector's technology. for the given capital and labour inputs, taking in to account depreciation, i.c..

$$
\begin{aligned}
& c_{i}(t)+\dot{K}_{i}(t) \leqslant F^{1}\left(L_{i}(t), K_{1 i}(t), \ldots\right. \\
& \left.K_{A_{1}}(t)\right)-\gamma_{i} K_{i}(t) \quad \text { for } i=1,3 \text { and } 4
\end{aligned}
$$

The housing sector $S_{2}$ has a similar equation, but with a different interpretation. since housing is not all consumed at one period and so cannot be considered a pure consumption good. Let $C_{2}$ represent a How. rather than a stock variable, $C_{2}(t)$ being the amount of housing added at time $t$ to the previous stock of housing. The corresponding stock variable is denoted $c_{2}$. Hence. consumption of sector $S_{2}$ at time $t$ is approximately represented by the integral of the difference between the accumulated housing produced and what is deprecrated until time $t$ :

$$
c_{2}(t)=\int_{0}^{t}\left(C_{2}(\xi)-r_{2} C_{2}(\xi)\right) d z
$$

The equation corresponding to equation (3) is:

$$
\begin{aligned}
& C_{2}(t)+\dot{K}_{2}(t) \leqslant F^{2}\left(L_{2}(t), K_{12}(t) \ldots . .\right. \\
& K_{42}(t)-\varkappa_{2} K_{2}(t) \quad(4)
\end{aligned}
$$

For each value of the population variable $L_{i}$, equations (3) and (4) give implicitly a choice between present and future consumption, when $F^{i}$ is an increasing function of the capital stock vector $\left(K_{1}, \ldots, K_{4 i}\right)$.

The initial data for the empirical model were 1960 values in US dollars. Estimations of the approximate initial values of consumption and investment in the four sectors, and of the production functions were carried out for each block. The production functions $F^{i}$ were assumed to be of the Cobb-Douglas type. i.e:

$$
\begin{aligned}
& F^{i}\left(L_{i}(t), K_{t i}(t) \ldots . K_{t_{i}}(t)\right) \\
& =\left(L_{i}(t)\right)^{x / 1} K_{1,}(t)^{\beta / t(t)} \times \ldots \times K_{4,}(t)^{f_{a}(t)}
\end{aligned}
$$

Hence, in the estimation. the values of the parameters $x_{1} \beta_{1}, \ldots, \beta_{4}$ were approximated. These types of production function were used because they allow some substitutions between factors: more general forms of production functions can be also used. Functions with fixed capital/labour ratios at each time period could alternatively be used provided that the ratios are allowed to vary through time. Initial values of capital labour ratios in the different sectors, denoted $K L_{j}, j=1, \ldots, 4$, were also estimated:

$$
K L_{i}(0)=\left(\frac{K_{L_{i}}(0)}{L_{i}(0)} \ldots \ldots \frac{K_{4 i}(0)}{L_{i}(0)}\right)
$$

with available data for each block of countries. They were used to determine bounds for the admissible changes in capital labour ratios. This constraint is formalized:

$$
\left.\left|\frac{\mathrm{d}}{\mathrm{d} t}\right| K L_{t}(t)\right)|| \leqslant Y_{i}
$$

equation (5) represents a limitation on the possible changes of capital labour ratios in each sector. 
Empirically, it was cstimated ${ }^{\mathbf{k}}$.

$$
\begin{aligned}
& Y_{1} \cong 0.1 \\
& Y_{2} \cong 0.05 \\
& Y_{3} \cong 0.05 \\
& Y_{4} \cong 0.1
\end{aligned}
$$

The growth of the economically active population was detcrmined from a demographic submodel. Basically, it was assumed that the rate of growth of the productive population depended on present and past consumption of the three basic goods, as well as present and past population and capital stock:

$$
\left.L(t)=\phi\left(c_{1}(\xi), c_{2}(\xi), c_{3}(\xi), l \xi\right), k(l)\right) \text { for } \xi \leqslant t
$$

The relation (6) was estimated with linear econometric techniques across countries and through time in the demographic empirical submodel.

In order to obtain a growth policy acceptable from a socio-economic viewpoint, the additional constraint is imposed:

$$
\frac{c_{d}(l)}{L(t)} \quad j=1, \ldots, 4
$$

are monotone nondecreasing functions of time, i.e. per capita consumption of basic goods is increasing, or nondecreasing through time for any feasible growth policy. Given that, as is generally assumed in optimal growth models, consumption in each period is smaller than or equal to total production in each sector, this constraint implicitly determines a lower bound on terminal values of per capita stock of capital in the different sectors when final minimum acceptable values of consumption are given. This is formalized in the next section.

In view of the above description of the economy, the problem may now be formalized as follows. To choose the instruments: consumption paths in sectors 1,2 , and 4 , and a path $C_{2}(t)$ of added housing stock represented by the vector:

$$
C(t)=\left(c_{1}(t), C_{2}(t), c_{3}(t), c_{4}(t)\right)
$$

and investment and factor allocation paths:

$$
\begin{aligned}
k(t) & =\left(K_{j i}(t)\right) & i, j & =1, \ldots, 4 \\
l(t) & =\left(L_{i}(t)\right) & i & =1, \ldots, 4
\end{aligned}
$$

as functions of time. so as to attain in the minimum possible time. acceptable consumption values of the first three sectors (nutrition. housing, health and education). An economy $E$ is identified by an initial capital stock vector in the different sectors $K_{0} \in R^{4+}$. initial productive labour value in the different sectors $L_{0} \in R^{-}$, initial vector of consumption of the four types of goods denoted $c_{0} \in R^{4+}$, a vector of continuous production functions of the sectors $F=\left(F^{1}, \ldots, F^{4}\right)$, a continuous function $\phi$ linking productive population growth with consumption of basic goods as in equation (6) above, and initial capital/labour ratios in the four sectors.

A feasible growth path for the economy $E$ is a triple

\footnotetext{
- The construnts effectively imposed on the empirical model are an approximation to equation $\$ 51$.
}

of consumption and capital and labour allocations through time:

$$
\{c(t), k(t) .\lfloor(t)\rangle
$$

where

$$
d t)=\left(c_{1}(t), c_{2}(t) \cdot c_{3}(t), c_{4}(t)\right)
$$

with initial values $c(0)=c_{0}, K(0)=\left(K_{1}(0), K_{2}(0), K_{3}(0)\right.$. $\left.K_{4}(0)\right)=K_{0}$, and $L(0)=L_{0}$, and satisfying the constraints above (equations $1-7$ ).

Given a vector of minimum acceptable per capita consumption of the three basic goods $c=\left\{c_{1}, c_{2}\right.$, $\left.c_{3}\right\} \in R^{3+}$. (nutrition, housing, health and education. respectively). an efficient growth path for the cconomy $E$ is a feasible growth path $(c *(t), k *(t),(*(t))$, such that c* attains or surpasses the minimum acceptable per capita levels of consumption $c$ at time $T$, and $T$ is the shortest time horizon in which $\boldsymbol{c}$ is attained, i.e.:

$$
\left(\frac{c_{1} *(T)}{L(T)}, \frac{c_{2} *(T)}{L(T)}, \frac{c_{3} *(T)}{L(T)}\right) \geqslant c
$$

and, if $(c, k, l)$ is any feasible path, then:

$$
\left(\frac{c_{1}(t)}{L(t)}, \frac{c_{2}(t)}{L(t)}, \frac{c_{3}(t)}{L(t)}\right) \geqslant c \quad \text { if }(t)<T
$$

In the Appendix a few remarks are made on the application of the theoretical model discussed above to the empirical model.

\section{Existence and characterization of solutions}

The model discussed in the previous section was intended as an analytical framework for the empirical model on to which data was fitted and interpreted. and simulation was performed to analyse the effect of alternative policies. In the following we shall study sufficient conditions for the existence of an optimal growth path. and discuss a characterization of the solution(s) of a special case of the model by a system of differential equations. It is of interest to characterize the efficient path(s) by adequate conditions since such a characterization can be used to compare the properties of this model with other models, to study properties of the solution(s). such as questions on convergence of myopic efficient paths to global ones, and to derive algorithms for approximation of the solutions ${ }^{1}$. The results given here are a step towards a solution, rather than a complete solution of the problem.

From a mathematical viewpoint the model can be thought of as a dynamical system evolving through time. At any moment $t$ the system is in some state. For

\footnotetext{
For existence of a solution we use the results in Chichilnisky ${ }^{4}$ Because of possible non-convexities and other characteristics of the model. the techniques of reference 4 seem preferable to those of optimal control theory here. Non-convexities can urise in the production functions idue to, for instance, increasing returns at some production levelst and to the population rate of growth functions. These techniques are also useful since we can show existence of solutions in Hilbert spaces ( $L_{2}$ and Sobolev's spaces). Together w:th the characterization of optumal paths by optimal control theory tectiniques w which is given here for a special case and under convexuty assumptionsl. the results can then be used for the approxumation of the solutions with gradient methods which are available in Haibert spaces.
} 
an economic system. the amount of capital goods of each sector $K_{i}, i=1 \ldots \ldots, 4$ and of labour, $L$ constitute a suitable state description. Hence the state space is $R^{5+}$

At any time $t$ there is a vector which can be chosen by a decision maker from a feasible set (which, in general. varies with time and on the state). This vector is usually referred to as a decision or control variable; in economics it is called an instrument. Hence, the instruments are the allocations of resources to different productive uses and to consumption, i.e.:

$$
\begin{aligned}
K^{i}(t) & =\left(K_{1}(t), \ldots, K_{4}(t)\right) \quad i=1, \ldots, 4 \\
k(t) & =\left\{K_{j}(t)\right\} \quad i=1, \ldots, 4, \quad j=1, \ldots, 4 . \\
l(t) & =\left(L_{1}(t), \ldots, L_{4}(t)\right)
\end{aligned}
$$

and

$$
c(t)=\left\{c_{1}(t), c_{2}(t), c_{3}(t), c_{4}(t)\right)
$$

The instruments are taken to vary within a feasible set which depends on the state and on time. In our casc. as discussed in this section:

$$
\begin{aligned}
& \sum_{i=1}^{4} K_{j}(t) \leqslant K_{i}(t) \quad K_{j}(t) \geqslant 0 \quad \text { for all } i, j \\
& \sum_{j=1}^{4} L_{i}(t) \leqslant L(t) \quad L_{j}(t) \geqslant 0 \quad \text { for all } i \\
& c_{i}(t) \geqslant 0 \quad \text { for all } i
\end{aligned}
$$

It is assumed that a value of the state and of the instrument variables at any point of time completely determine the dynamics of the system. Thus, for a given technology the capital and labour structure with its allocation among different uses (as given by the instruments) determines the output of all goods and their allocation between consumption and investment. In view of the formulation of the first section. the evolution of the state of the system is thus given by the equations:

$$
\begin{aligned}
\dot{K}_{i}(t)= & F^{i}\left(L_{1}(t), K_{1}(t), \ldots, K_{4 i}(t), t\right)-c_{i}(t) \\
& -r_{i} K_{i}(t), \quad i=1,3 \text { and } 4 \\
\hat{K}_{2}(t)= & F^{2}\left(L_{2}(t), K_{12}(t), \ldots, K_{42}(t), t\right)-C_{2}(t) \\
- & \dddot{r}_{2} K^{2}(t)
\end{aligned}
$$

and

$$
L(t)=\phi\left(c_{1}(t), c_{2}(t), c_{3}(t), L_{1}(t), \ldots, L_{4}(t), k(t), t\right)
$$

(where the dot denotes time derivative) provided that equation $(6)$ is simplified to assume that the effect of past consumption on population growth at time $l$ is embodied by $\phi$ in equation (10); otherwise. the system becomes an infinite dimension dynamical system and requires more complicated mathematical tools. The time variable t enters into $F^{i}$ and $\phi$ to allow $F^{i}$ to vary over time. due to technological progress or other exogenous factors.

We then have a dynamical system on the state space $R^{j+}$ depending on the parameters or instruments

$$
\left.f(t)=\left(K^{2}(t) \ldots . K^{4}(t)\right) \quad(t)=L_{1}(t) \ldots . . L_{4}(t)\right)
$$

and

$$
c(t)=\left(c_{1}(t), c_{2}(t), c_{3}(t), c_{4}(t)\right)
$$

The initial conditions of consumption, labour force and capital in the different sectors are:

$$
\begin{aligned}
& C(0)=C_{0} \\
& L(0)=L_{0} \\
& K(0)=K_{0}
\end{aligned}
$$

a specification of technology $F^{i}$ and of population rate of growth relation $\phi$. The last two constraints of the model are that the vector valued function:

$$
\frac{c(t)}{L(t)}
$$

be monotone nondecreasing through time, and:

$$
\left\|\frac{\mathrm{d}}{\mathrm{d} t}\left(K L_{i}\right)\right\| \leqslant Y_{i} \quad i=1, \ldots, 4
$$

as in equation (5). A feasible growth path can now be thought of as a choice of instruments through time $(C(t), l(t), k(t))$ satisfying the initial conditions (equation 11), and the relations (8), (9), (10). (12) and (13).

Let $G$ be the translation of the positive cone $R^{3+}$ with vertex $c=\left(c_{2}, c_{2}, c_{3}\right)$ denoting minimum acceptable per capita levels of consumption of the three basic goods. Then. the problem can be formulated as minimizing the time for reaching the set $G$, i.e., to find a feasible growth path such that there exists a time horizon $t_{0}$, with:

$$
\left(c_{1}(t) / L(t), c_{2}(t) / L(t), c_{3}(t) / L(t)\right) \in G \quad \text { for } t \geqslant t_{0}
$$

and such that if $(c t), l(t), k(t))$ is another feasible growth path with:

$$
\left(c_{1}\left(t_{1}\right) / L\left(t_{1}\right), \ldots, c_{3}\left(t_{1}\right) / L\left(t_{1}\right)\right) \in G, \quad \text { then } t_{1} \geq t_{0} \text {. }
$$

The first problem one faces in attempting to prove the existence of a solution is that the set $G$ may not be reachable from the initial conditions (equation 11) through feasible paths of the system described by equations (8) (13). One can at most expect to find sufficient conditions on the system for the set $G$ to be reachable. Initial conditions (equation 11 ) and the relations $(8),(9)$ and $(10)$ determine a typical control problem (without the additional restrictions of equations (12) and (13)). In many simple cases it is easy to see which points in the state space are reachable and which are not for this type of system: the linear case, for instance. has been treated in detail by LaSalle ${ }^{2}$ and Boltyanskii et al, ${ }^{2}$ Equations (12) and (13). and the fact that the region to be reached is one in the variable $\left(c_{1} / L, c_{2} / L\right.$ and $\left.c_{3} / L\right)$ which is a combination of instrument and control variables make our problem more complex: further study of mathematical conditions on the system for reachability of the set $G$ are required. These conditions should be adequately interpreted in functions of the technologies. of the population growth equations. etc. to be meaningful for the economic model.

Assumprion 0 . In what follows we assume that there exists a $T_{0}<x$ and a feasible growth path $(a t)$. $(t)$. $k(t))$. such that

$$
\left(c_{1}(t): L(t), c_{3}(t): L(t) \cdot c_{3}(t) L(t)\right) \in G \quad \text { for } t \geqslant F_{0}
$$


In order to show the existence of an efficient path or solution we make a few assumptions and discuss the spaces of growth paths we shall consider. Let $F(l, k, t)$ be the vector valued function:

$$
F(i, k, f): R^{4+} \times R^{16} \times R^{+} \rightarrow R^{4+}
$$

given by

$$
\begin{array}{r}
F(1, k, t)=\left\{F^{1}\left(L_{1}, K_{11}, \ldots, K_{41}, t\right), \ldots\right. \\
F^{4}\left(L_{4}, K_{1, \ldots}, \ldots L_{44}, t\right)
\end{array}
$$

where for each $i$,

$$
F^{i}\left(L_{i}, K^{i}, t\right): R^{6+} \rightarrow R^{-}
$$

is the function representing the technology of sector $i$ at time $t$.

We make the following assumptions about the technology:

Assumption 1: $F$ admits an extension to a real valued function $F^{1}$ defined on a neighbourhood of $R^{4+} \times R^{16+} \times R^{*}, F^{1}$ continuously differentiable and nondecreasing, and for all $i$ there exists $\bar{K}^{i}$ with $F^{i}\left(L_{i}\right.$, $\left.K^{i}, t\right)<j_{i} K_{i}$ for any $L_{i}, t$, if $K^{i}>R^{i}$, and $K_{i 0}<\sum_{j=1}^{4} \bar{K}_{i j}$ (where $\bar{K}^{i}=\left(\bar{K}_{\mathrm{t} i} \ldots \ldots, \bar{K}_{4 i}\right)$, and where $K_{0}=\left(\widetilde{K}_{10}, \ldots ., K_{40}\right)$ are the initial values $)$. This assumption is basically a technological constraint on productivity: it formalizes the notion that after certain levels of capital stocks the technology is constrained in its increases on productivity by the cost of maintaining capital stocks at operating levels (represented by the depreciation parameter ri). $^{2}$.

We make the following assumptions about the population growth.

Assumption 2: The function $\phi: R^{25+} \rightarrow R^{+}$admits an extension to a real-valued function $\phi^{\prime}$ defined on a neighbourhood of $R^{25+}, \phi^{\prime}$ continuously differentiable. and $|\phi(c,-)| \leqslant K$ for some $K>0$. This assumption formalizes the notion that the rate of population growth is bounded in absolute value. This hypothesis can be derived from others, for instance, that after certain levels of consumption, the rate of growth of the population is nonincreasing. or another weaker hypothesis,

Let $T$ be a real number, $T>T_{0}$.

We define the set $\mathbb{C} \times \mathbb{L} \times \mathbb{K}$ of all feasible consumption, labour, and capital allocation paths as follows ${ }^{\mathrm{m}}$ :

(at). $(t), k(t)) \in \mathbb{C} \times \mathbf{L} \times \mathbb{K} \quad$ if and only if:

(i) $c_{1}(t), c_{3}(t)$ and $c_{4}(t)$ are in $\left(L_{2}[0, T]\right)^{+}+c_{2}(t)$ in $\left(H_{2}^{1}\right)^{*}, l(t) \in\left(H_{2}^{1}[0, T]\right)^{4+}$ and $k(t) \in\left(H_{2}^{1}[0, T)^{16+}\right.$

(ii) Constraints (equations $8-13$ ) are satisfied a.e. on $c_{i}(t)$ in $\left.0, T\right), i=1.3$ and 4 and for all

\footnotetext{
$\mathrm{m}$ In the following. $L_{2}[0, T]$ denotes the Hilbert space of measurable

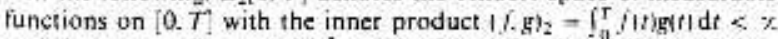
and the norm $\mid f \|_{2}=\left(f,\left.f\right|_{2} ^{1}{ }^{2}, H_{2}^{t}[0 . T]\right.$ is defined as tollows: if $f . g$ are in $C_{h}^{t}$ icontinuousty differentiable/ functions on $0 . T$. define the inner product:

$$
(f, g)_{2}^{1}=\int_{11}^{j} \sum_{0 x+2<1} D^{\prime} f(z)-D^{\prime} R(n) \mathrm{dr}
$$

where $D^{\mu}$ denotes the $j$-derivative operator. $D^{\prime \prime}(j)=f$. Let the norm 1.1 be detined as $\left.\|f\|_{2}^{1}=11,1, f 1,\right\}^{2}$. The completion of $C_{1}^{1}$ under this norm is a Hilbert space. called a Sobolev's space denoted $H_{2}^{1} 0 . T$. By Soholev's theorem ${ }^{8} H_{2}[0 . \mathrm{r}]=\mathrm{C}_{0}[0 . I]$. 1.e.. all functiuns in $H_{2}^{1}$ are continuous
}

$t \in[0, T]$ for $\pi(t)$ and $k(t)$ and $c_{2}(t)$.

(iii) $0 \leqslant c_{1}(t) \leqslant F^{t}([(t), k(t), f)$ for $i=1,3,4$ and $0 \leqslant C_{2}(t) \leqslant F^{2}(i)(n, k(t), l)$ a.e. in $[0, T]$. (In other words. consumption of sectors $S_{1}, S_{3}$, and $S_{4}$ and output of housing $\left(C_{2}\right)$ in each period at each sector cannot exceed that sector's total output.) (iv) $\left\{c^{\prime}(t) L L(t), c_{2}(t): L(t), c_{3}(t), L(t)\right\} \in G$ a.e, in $\left[T_{0}, T\right]$.

Note that assumption (iii) together with (iv) and equation (12) above imply that the feasible terminal values of the capital stock vector:

$$
K(T)=\left(K_{1}(T) \ldots, K_{4}(T)\right)
$$

are bounded from below. Not all capital stock can be consumed near the end of the growth path since, otherwise, production and hence consumption. by (iii) would drop beneath the required levels (assumption iv), which are sustained (by equation 12). In view of the assumptions, the following result now follows from Theorem 1 (reference 4):

Theorem 1: For any economy $E$ satisfying assumptions 0.1 and 2 there exists an optimal growth path $(c *, l *, k *)$ in the set of feasible growth paths $\mathbb{C}$ $\times \mathbb{L} \times \mathbb{K}$.

Under certain conditions, mostly convexity assumptions on the technology $F$ and the function $\phi$ giving the rate of population growth. one can also, prove the uniqueness of the consumption path $c *$. obtained from a solution, as in Theorem 1 (reference 4). Note that since the space of instruments has a dimension larger than 5 , and all that is needed to fully determine the dynamical system is to give a vector in the tangent space for each point of the state space $R^{s+}$. one can expect that more than one path of instruments will yield the same solution. This could be used for studying possible alternative optimal policy decisions.

We next discuss a characterization for the solution(s). In order to use optimal control theory techniques (see, for instance, Roxin ${ }^{9}$ ), we make strong assumptions and simplifications in the model. In (Assumption 5) below. we make convexity assumptions which are generally required for the characterization of optimal paths in optimal control theory. Further, we assume that the problem of reaching the set $G$ (defined above) in a minimum time can be given as one of minimization of the time needed to reach a set in the state space. where the values of the state variables. labour and capital allocation to the different sectors are such that they make possible sustained per cupita consumption of the minimum acceptable per capita consumption values denoted by $c$ above".

\footnotetext{
"This last assumption will usually be satisfied in one sector models. but may not be satisfied in general. The puint is that in it multisector model. if $\left\{c *, k_{*},\{*\}\right.$ is a path thit minimizes the time needed isay $T$, to reach a set $G$ where the final value of the variabies $C_{1}: L$. $C_{2} L$ and $c_{3}: L$ are above is ralue $c$, and if $I K_{1}, \ldots, K_{4} * L * 1$ is the corresponding eapural stock and labour parh in the state space, then it is not necessarily true that $\left(K, x \ldots \ldots, K_{4}, L, *\right)$ minimizes under the same constraints the time required to reach $\left.\left\{K_{1}, \mid T\right\} \ldots, K_{4}=1\right\}$. L-1. Economically. this means that planning it minimum time path to reach the productive capabilities or values of inputs shat would allow one to produce centain levels of ger cumas consumpsion may nol coincide with planning a minimum time path to reach those leveis of per tapira consumption. This is a point of posstbly interestung policy implications and which requires further andlysis.
} 
Economic deveio wroung and efficisncy criteria in satisfaction of basic needs: $G$. Chichilnisky

We now rewrite the simplified version of the model in optimal control notation. Since the characterization we seek is for medium- or short-term comparisons with other planning policies. we shall assume a certain given rate of population growth la particular example. used for instance. in reference 4 is: $L(t)=e^{j \prime} L(0)$, $i>0$ ). Hence. in the following, the differential equation corresponding to population growth (equation 10) will not be part of the system. the production functions will be assumed homogeneous. and variables are to be understood in per capita terms from here on fi.e., divided by the total population variable $L(t)$, for each $t)$; we preserve the same notation to simplify the exposition. For simplicity, the vector $c_{2}$ $+K_{2}$ will now be denoted $K_{2}$, since, as discussed in the section on the model and its dynamics, $c_{2}$ is a stock variable. Hence, here the minimum acceptable level of housing and capital stock in $S_{2}$ becomes $c_{2}$ $+K_{2}$. where $c_{2}$ is the minimum level of housing stock of above, and $\boldsymbol{K}_{2}$ is the value of the stock variable associated to $c_{2}$ in equation (9).

Let $\dot{x}$ denote the vector of state variables $\hat{x}=\left(K_{1}, \ldots, K_{4}\right) \in \mathbb{R}^{4+}$ and let $x$ denote the vector $(t, x) \in R^{5 *}$. These vectors are considered in Fuclidean spaces with the usual norms. Let $u$ denote the instrument vector:

$$
\left.u=(c, l, k)=i c_{1}, c_{3}, c_{4}, L_{1}, \ldots, L_{4}, K^{1}, \ldots, K^{4}\right)
$$

$c$ here denotes the vector $\left(c_{1}, c_{3}, c_{4}\right)$. Translated to this case. the constraints (8) and (11) determine the feasible set of instrument vectors $u$, as depending on time and on the state, denoted $U(x, t)$. As seen in the proof of Theorem $1^{4}$, and in view of assumptions $1-4$. $U(x, t) \subset U$. a compact set of the $u$-space.

We can now rewrite the system (9) and (10) as:

$$
\dot{x}=f(t, x, u) \quad \text { with } \quad x(0)=x_{0}
$$

or

$$
\vec{r}_{1}=f^{i}(x, u) \quad i=1 \ldots .5
$$

where

$$
\begin{aligned}
& \hat{x}_{1}=1 \quad x_{2}=K_{1} \ldots, \quad x_{5}=K_{4} \\
& u=(c, I, k) \\
& f^{\prime}=1 \\
& f^{2}=F^{1}\left(L_{1}, K_{11} \ldots . K_{41}\right)-c_{1}-\gamma_{1} K_{1} \\
& f^{3}=F^{2}\left(L_{2}, K_{12} \ldots, K_{42}\right)-\because_{2} K_{2} \\
& f^{4}=F^{3}\left(L_{3}, K_{1}, \ldots . . K_{4,3}\right)-c_{3}-\dddot{;}_{3} K_{3} \\
& f^{5}=F^{4}\left(L_{4}, K_{14}, \ldots, K_{44}\right)-c_{4}-7_{4} K_{4}
\end{aligned}
$$

As before:

$$
\sum_{j=1}^{4} \mathrm{~K}_{j i}(t)=\mathrm{K}_{i}(t)
$$

and since we are now rewriting the system in per capita terms:

$$
\sum_{i=1}^{4} L_{i}(t) \equiv 1
$$

We now make further assumptions.

Assumprion 5: the sets:

$$
F(x, U)=\left\{F(x, u, t): u \in U_{i}\right.
$$

and

$$
\phi(x, v)=\left\{\phi(x, u, t): u \in U_{1}\right.
$$

are both convex.

Note that since $U$ is compact, and $F$ and $\phi$ continuous these sets are compact too. Convexity of these sets can be obtained from concavity of the technology $F$ and of the function of population rate of growth $\phi$ (see reference 4 ).

4ssumption 6: Let $(c * . ; *, k *)$, be an efficient path and $\left(K_{1} * \ldots, K_{4} *\right)$ be the associated path of state vectors.

If $T$ is the minimum time at which $\left\langle c_{1} * c_{2} *\right.$, $c_{3} * t \geqslant c$ a.c. for $t \geqslant T$, then assume that $T$ is also the minimum time at which any feasible path $\mid K_{1}(r)$. $\left.\ldots, K_{4}(t)\right)$ reaches the set:

$$
\hat{H}=\left\{x \in R^{s+}: \hat{x} \geqslant\left(K_{1} *(\bar{l}), \ldots, K_{4} *(\bar{t}) ;\right.\right.
$$

(see footnote n). Assume, further, that $\hat{A}$ is reachable from the initial conditions. Then we obtain:

Proposition 2: Under Assumptions 5 and 6 if $u *=\left\{c *, k *, I_{*}\right\}$ is an efficient growth path, then there exists a vector valued function of time $p(t)$ in $\left(H_{2}^{1}\right)^{5}$ such that $p(i)$ satisfies the differential equation ${ }^{\circ}$

$$
\dot{p}=-p\left(\frac{\partial f}{\partial x}\right)
$$

at $u *=(c *, / *, k *)$ and the corresponding $x_{*}=\left(t, K_{1} *, \ldots, K_{4} *\right)$, and so that. at each time $t$. $u *=(c *(t), l *(t), k *(t))$ maximizes the scalar product

$$
p(t) \cdot f(x *(t), \tan (t))
$$

with respect to all possible values of $u$ in the set $U$.

Proof: By Assumptions 5 and 6 we can apply the Pontryagin maximum principle to the problem of system (15) reaching in minimum time the set $\dot{H}$ in the state space. which proves the existence of a $p(t)$ satisfying equation (17), with wrie $R^{s+}$ for each $t$. By definition of the space of paths in the state space, p(f) $\in\left(H_{2}^{1}\right)^{5 *}=\left(H_{2}^{1}\right)^{5}$ since $\left(H_{2}^{1}\right)^{5}$ is a Hilbert space.

Equation $(16)$ then follows from usual optimal control theorems ${ }^{2.5}$

\section{Conclusions}

(1) Under the assumptions, Proposition 2 gives a characterization ${ }^{p}$ of the optimal path of instruments $u *(t)$ as one maximizing, for each time $t$, the scalar product:

$$
p(t) \cdot f(x(t), u(t))
$$

for all feasible $1(t)$ in $U$, where $p(t)$ satisfies equation (16) above.

\footnotetext{
"At the points $54(t)$ where the houndary of the reachable set of the system $\mid 15\}$ (sce, for instance Roxin ${ }^{\dagger}$ is a smooth surface.

eNote that in order to compietely determine the paths pa1t). one needs ro give in addition to Proposition 2. a sct of final condations of $p+1 T_{1}$, alsi cilled transversality conditions, representing the constraint that the etficient path reaches $\dot{H}$ al.tume $T$, and hence. depending on the properties of the set $H$. For discussions and reference to these and other aspects of the determanation of Iransversality conditions in minimum time problerns, sec feference
II.
} 


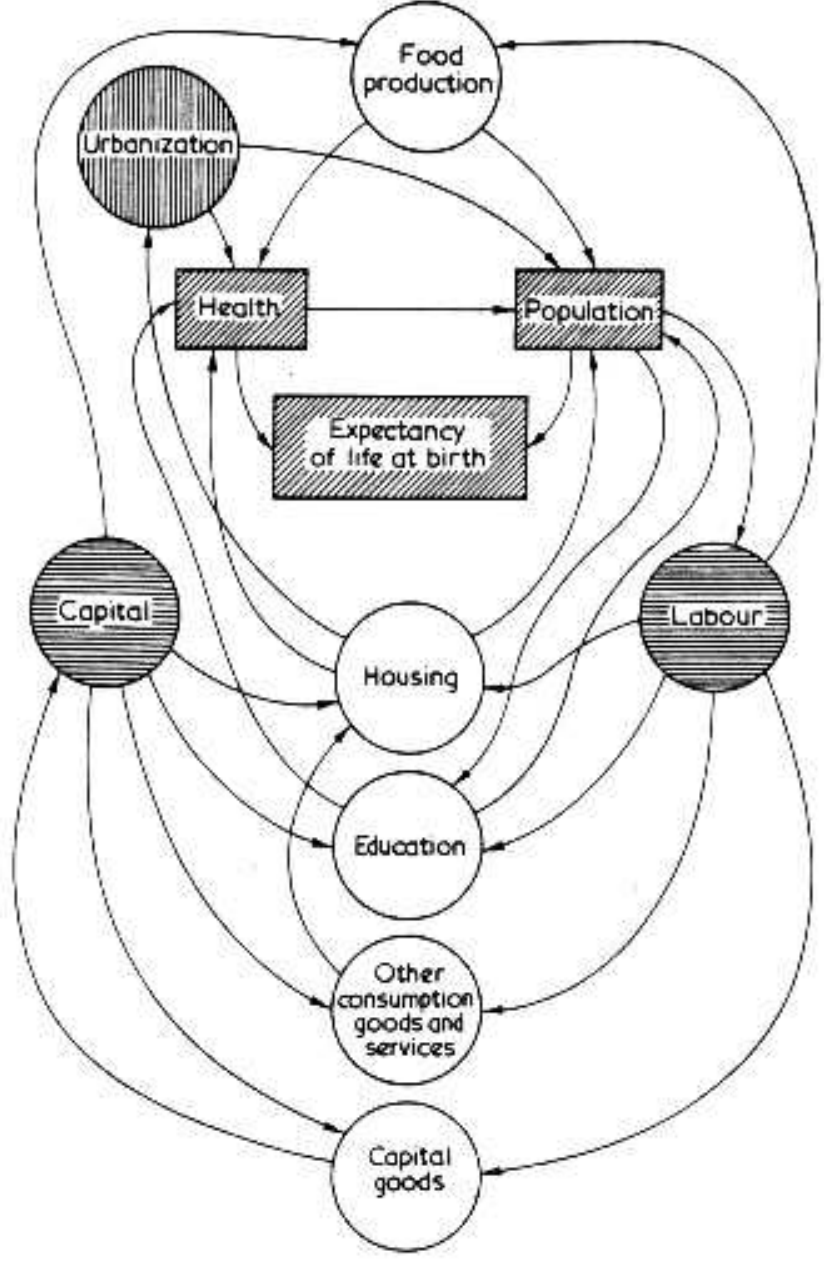

Figure f Graphical representation of model

(4) The data bank of the model was built using essentially compilations of specialized agencies of the United Nations - particularly the Yearbooks on demography, statistics and National Accounts-and information from OIT, UNESCO, and FAO.

Much information was compiled. However, the present available data is far from being satisfactory. As a result, some subjective judgment is unavoidable in the process of covering gaps in information. This is also true for the selection of available data. Tests were made with the model to verify the sensitivity of the model to variation on the data observed or used; the model and its results proved to be reasonably stable.

(5) The variables describing evolution of the population life expectancy at birth and death rates and of the number of family members are functions of socio-economic variables. The main difference between this demographic model and those of others in use is that it is not projective, it does not extrapolate observed tendencies, but it relates them to socioeconomic variables.

A large number of tests were carried out until the functional relations connecting the variables were in agreement with the data and satisfied econometric tests (see Demographic sector ${ }^{6}$ ). However, demographic data prior to 1950 is quite incomplete.

(6) The global dynamics of the empirical model are suggested graphically in Figure 7 . The initial data on economically active population. activity rates, and stock of capital determine in each period the production levels of the five sectors of the model. Actual data are used for the $t=0$ period; the values for $t>0$ arise from the combination of the initial conditions and of the constrained optimization process.

The level of physical production of food, housing and health and education services, divided by the population. determines the levels of satisfaction of basic needs.

Given the initial yalues. the yearly housing investment determines the urbanization rate, as it is assumed that all inversions in this sector are assigned to urban type housing (see Urbanization and housing ${ }^{6}$ ).

Figure I represents a sequential (i.e., discrete time) version of the model as was simulated in the computer work. Labour and capital, through the production process yield values of food, housing, education, of her goods and services and capital goods production. Food, education and housing, in turn, affect health and urbanization variables. Health is also affected by urbanization. Health, urbanization, housing and food in turn feed population variables. New capital and population variables thus determined, give the value of the total productive factors (capital and labour) available for the next period. completing the cycle.

The expectancy of life at birth, (ELB) variable which is in the centre of the diagram was not considered in the theoretical model. It was chosen. however. in the computational model as a proxy of social welfare or level of satusfaction of basic needs; this was done for practical purposes since ELB appears to be a good indicator ${ }^{6}$. Because of the complex nature of the optimization model. the large number of variables and the amount of data needed, the computation of optimal paths was redefined in the empirical model. The optimal paths were computed 'myopically': at each period the allocation of factors in the different sectors was chosen so as to maximize ELB in the next period, with constraints in the investment at that period. Time periods were one year long. This procedure, of course, raises questions about the relation between global and myopic optimization results. This is one point in which the theoretical model presented here and the empirical model are different; the two problems do not reduce to a common formulation. 